\section{Case Reports in Ophthalmology}

Case Rep Ophthalmol 2020;11:256-262

DOI: $10.1159 / 000508389$

Published online: July 1, 2020
(C) 2020 The Author(s)

Published by S. Karger AG, Basel www.karger.com/cop

\title{
A Case of Fungal Endophthalmitis Caused by Paecilomyces lilacinus that Might Have Spread from the Sclera into the Intraocular Space
}

\author{
Akiko Tara $^{a}$ Takatoshi Kobayashia ${ }^{a}$ Ryohsuke Kohmoto ${ }^{a}$ \\ Masanori Fukumoto ${ }^{a}$ Takaki Sato $^{\text {a }}$ Teruyo Kida $^{a}$ Yukimasa Ooi ${ }^{b}$ \\ Tsunehiko Ikeda ${ }^{\mathrm{a}}$

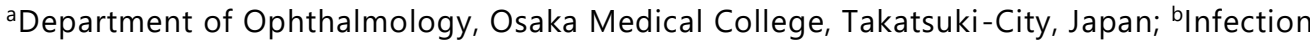 \\ Control Office, Osaka Medical College, Takatsuki-City, Japan
}

\section{Keywords}

Fungal endophthalmitis · Paecilomyces lilacinus · Conjunctival abscess · Vitrectomy

\begin{abstract}
The aim of this report was to describe a case of fungal endophthalmitis possibly caused by Paecilomyces lilacinus (PL) penetrating the sclera from a conjunctival abscess. This case study involved an 83-year-old male patient with a past history of scleral buckling, subtenon steroid injection, and cataract surgery. The vitreous opacity and a conjunctival abscess appeared in the inferonasal quadrant of his right eye at 5 months after cataract surgery. PL was isolated from a cultured conjunctival discharge specimen obtained from the patient's right eye. Although the treatment with antifungal agents relieved the conjunctival abscess, the vitreous opacity became worse. Thus, vitrectomy was subsequently performed. Intraoperative findings revealed severe vitreous opacity in the inferonasal quadrant, adjacent to the sclera at the site of the conjunctival abscess. Our findings indicate that fungal endophthalmitis appeared to have been caused by PL in the conjunctival abscess that may have penetrated the sclera and spread into the intraocular space.




\section{Case Reports in Ophthalmology}

Tara et al.: Fungal Endophthalmitis Caused by Paecilomyces lilacinus

\section{Introduction}

Paecilomyces lilacinus (PL) is a filamentous fungus that is widely distributed in both soil and air, and although minimally non-pathogenic, it reportedly is a causative agent for endocarditis, pyelonephritis, sinusitis, and dermatitis, etc., in immunocompromised patients [1]. In the field of ophthalmology, keratitis is a frequently reported infectious disease caused by PL [2-5], and there are also sporadic reports of endophthalmitis caused by this fungus [6-10]. However, in the majority of such cases, PL reportedly spreads from the site of the trauma or surgical wound into the intraocular space. Here, we report a unique case of fungal endophthalmitis in which the disease was caused by PL that had possibly penetrated the sclera from a conjunctival abscess.

\section{Case Presentation}

An 83-year-old male was referred to our department for detailed examination and treatment after vitreous opacity was observed in his right eye at a neighborhood clinic. His past history included undergoing scleral buckling for rhegmatogenous retinal detachment in the superotemporal quadrant of his right eye in 1992. Moreover, in both January and April 2015, he had undergone posterior subtenon injection of triamcinolone for the treatment of diabetic macular edema in his right eye, and in June 2015, cataract surgery (phacoemulsification + intraocular lens implantation) was performed on that eye. In November 2015, the patient visited the above-noted neighborhood clinic after becoming aware of blurred vision in his right eye.

Upon initial presentation at our department, we noted that the patient was currently receiving outpatient treatment for diabetes mellitus (hemoglobin A1c: $7.0 \%$ ) and hypertension at the neighborhood clinic, and that there were no remarkable familial histories. Clinical examination revealed that his visual acuity was $\left(0.15 \times \mathrm{S}+1.00 \mathrm{D}=\mathrm{C}-3.00 \mathrm{DA} \times 90^{\circ}\right)$ OD and $\left(0.45 \times \mathrm{S}+1.00 \mathrm{D}=\mathrm{C}-3.00 \mathrm{DA} \times 100^{\circ}\right) \mathrm{OS}$, and that the intraocular pressure in his right and left eye was $11 \mathrm{~mm} \mathrm{Hg}$ and $10 \mathrm{~mm} \mathrm{Hg}$, respectively. Slit-lamp microscopy examination revealed a conjunctival abscess and inflammatory anterior chamber cells in the inferonasal quadrant of his right eye (Fig. 1). Funduscopy examination of that eye revealed vitreous opacity, which was severe in the inferonasal quadrant. Scleral buckling resulted in a scar formation around the superotemporal quadrant. Subsequently, pus was spontaneously discharged from the conjunctival abscess of the right eye, and the filamentous fungus PL was isolated from cultured discharge (Fig. 2). For treatment, pimaricin ophthalmic ointment and $1 \%$ voriconazole eye drops were applied, resulting in nearly complete disappearance of the conjunctival abscess 1 month later. However, the vitreous opacity was not resolved, and in fact, gradually worsened (Fig. 3). Therefore, vitrectomy was subsequently performed.

Intraoperative findings revealed that the vitreous opacity in his right eye was severe mainly around the inferonasal quadrant and adjacent to the sclera at the site of the conjunctival abscess (Fig. 4). Due to the fact that posterior vitreous detachment had already occurred and was accompanied by epiretinal membrane, it was peeled off with vitreous forceps. Although mild retinal vasculitis was intraoperatively observed mainly around the inferonasal quadrant, there was no evidence of retinal necrosis or retinal edema. In order to examine the status of the sclera, the conjunctiva was incised at the nasal side, and although the blood vessels in the sclera at this site were swollen, no findings such as apparent scleral melting and thinning were observed. Since the previously performed scleral buckling was confined to the 


\section{Case Reports in Ophthalmology}

Case Rep Ophthalmol 2020;11:256-262

DOI: $10.1159 / 000508389$

C 2020 The Author(s). Published by S. Karger AG, Basel www.karger.com/cop

Tara et al.: Fungal Endophthalmitis Caused by Paecilomyces lilacinus

superotemporal quadrant, no incisional wound was found in the inferonasal quadrant. Postoperatively, the visibility of the ocular fundus recovered, and the corrected visual acuity was improved to 0.7 (Fig. 5). PL was not isolated from the cultured vitreous humor that had been intraoperatively collected. Since then, and as of March 2019, the patient's symptoms have been stable, and he has experienced no recurrence.

\section{Discussion}

In the field of ophthalmology, the reported cases of PL-induced infectious disease include keratitis [2-4] and endophthalmitis [5-10], both of which have been increasing in recent years. The majority of such cases occur in elderly patients aged 70 years and older. In general, many patients infected with PL have concomitant diabetes mellitus. A history of steroid therapy is also a risk factor for infection. Endophthalmitis caused by PL is commonly induced by trauma and ophthalmic surgery, and is often difficult to differentiate from bacterial endophthalmitis occurring in the late-term period after intraocular surgery. In the late 1970s, repeated cases of PL-induced endophthalmitis were reported in association with cataract surgery, thus suggesting that contaminated intraocular lenses, themselves, were the potential cause of the disease [11, 12]. In 2004, Tarkkanen et al. [13] reported a case of PL-induced endophthalmitis after cataract surgery in which the infection route was unknown. In that study, the patient developed uveitis of unknown cause at 4 months after small-incision phacoemulsification, and PL was eventually isolated from the intraocular fluid.

As for the infection route in this present case, our patient had no history of intraocular surgery, yet had undergone scleral buckling surgery followed by subtenon steroid injection for diabetic macular edema. Thus, those surgical procedures might have induced infection. However, both the scleral buckling surgery and the subtenon injection were performed in the superotemporal quadrant, which differs from the site of the present lesion. The incisional wound of cataract surgery was also located in the superior quadrant, which is the common incision site. No lesions, such as conjunctival abscess, were found in this site. Thus, it appears unlikely that the scleral buckling and cataract surgery wounds were the PL entry point.

It should be noted that in the present case, the conjunctival abscess in the patient's right eye occurred in the inferonasal quadrant, the area in which vitreous opacity was also severe. Intraoperatively, no signs of apparent scleral necrosis or other conditions were found in the sclera at that same site, and there was also no evidence of retinal necrosis or retinal edema in the ocular fundus. However, the conjunctival abscess and the opaque portion of the vitreous body were situated adjacent to each other, thus suggesting that PL causing the abscess might have penetrated the sclera and spread into the intraocular space. The possible reasons for why PL was not isolated in the cultured vitreous body are that antifungal agents had been administered prior to culture, and that the central portion of the vitreous cavity was relatively less opaque and contained a low volume of PL.

Generally, the sclera is opaque and is protected by the outer layer containing fibrous collagen and elastic fibers. It is rare that bacteria penetrates the sclera and invades the intraocular space. However, a previous study reported a case in which Pseudomonas aeruginosa formed infectious foci on the sclera, produced collagenase with which the sclera was dissolved, and subsequently spread into the intraocular space to cause endophthalmitis [14]. Despite the differences between bacteria and fungi, PL also sometimes forms infectious foci on the sclera, as P. aeruginosa. There have been previously reported cases in which PL infection reaching the deep scleral layer was resolved by sclerectomy $[15,16]$. Martínez et al. [17] 


\section{Case Reports in Ophthalmology}

Tara et al.: Fungal Endophthalmitis Caused by Paecilomyces lilacinus

reported that keratinase isolated from PL has high enzyme activities against collagen. Narita et al. [15] reported a case in which partial sclerectomy was effective for PL-induced scleritis, and Yoshida et al. [18] reported a case in which Purpureocillium lilacinum, the same genus as PL, penetrated the sclera and caused endophthalmitis [19].

In our present case, although neither apparent scleral necrosis nor thinning was observed in the sclera in the inferonasal quadrant where the conjunctival abscess occurred, the possibility of microscopically undetectable fungal infiltration into the sclera cannot be ruled out. We believe that patients with conjunctival abscesses caused by PL should be followed up while keeping in mind the possibility of the development of endophthalmitis.

\section{Acknowledgements}

The authors wish to thank John Bush for editing the manuscript.

\section{Statement of Ethics}

The protocols of this study were approved by the Ethics Committee of Osaka Medical College, Takatsuki-City, Osaka, Japan. In accordance with the tenets set forth in the Declaration of Helsinki, prior written informed consent was obtained from the patient.

\section{Disclosure Statement}

There are no conflicts of interest to report for all authors.

\section{Author Contributions}

A.T. and T.K.: equal contribution of patient management, conception of the paper, data analysis and interpretation, manuscript drafting, and literature search. R.K., M.F., T.S., T.K., and Y.O.: co-writing of the manuscript, literature search. T.I.: design of the paper, co-writing of the manuscript, literature search, manuscript editing and revision as well as final approval. All authors read and approved the final manuscript.

\section{References}

1 Orth B, Frei R, Itin PH, Rinaldi MG, Speck B, Gratwohl A, et al. Outbreak of invasive mycoses caused by Paecilomyces lilacinus from a contaminated skin lotion. Ann Intern Med. 1996 Nov;125(10):799-806.

2 Sharma V, Angrup A, Panwar P, Verma S, Singh D, Kanga A. Keratitis by Paecilomyces lilacinus: A case report from Sub-Himalayan region. Indian J Med Microbiol. 2015 Oct-Dec;33(4):585-7.

3 McLintock CA, Lee GA, Atkinson G. Management of recurrent Paecilomyces lilacinus keratitis. Clin Exp Optom. 2013 May;96(3):343-5.

4 Deng SX, Kamal KM, Hollander DA. The use of voriconazole in the management of post-penetrating keratoplasty Paecilomyces keratitis. J Ocul Pharmacol Ther. 2009 Apr;25(2):175-7.

5 Chakrabarti A, Shivaprakash MR, Singh R, Tarai B, George VK, Fomda BA, et al. Fungal endophthalmitis: fourteen years' experience from a center in India. Retina. 2008 Nov-Dec;28(10):1400-7.

6 Gupta A, Srinivasan R, Kaliaperumal S, Saha I. Post-traumatic fungal endophthalmitis-a prospective study. Eye (Lond). 2008 Jan;22(1):13-7. 


\section{Case Reports in Ophthalmology}

7 Garbino J, Ondrusova A, Baglivo E, Lew D, Bouchuiguir-Wafa K, Rohner P. Successful treatment of Paecilomyces lilacinus endophthalmitis with voriconazole. Scand J Infect Dis. 2002;34(9):701-3.

8 Domniz Y, Lawless M, Sutton GL, Rogers CM, Meagher LJ. Successful treatment of Paecilomyces lilacinus endophthalmitis after foreign body trauma to the cornea. Cornea. 2001 Jan;20(1):109-11.

9 Okhravi N, Dart JK, Towler HM, Lightman S. Paecilomyces lilacinus endophthalmitis with secondary keratitis: a case report and literature review. Arch Ophthalmol. 1997 Oct;115(10):1320-4.

10 Levin PS, Beebe WE, Abbott RL. Successful treatment of Paecilomyces lilacinus endophthalmitis following cataract extraction with intraocular lens implantation. Ophthalmic Surg. 1987 Mar;18(3):217-9.

11 Mosier MA, Lusk B, Pettit TH, Howard DH, Rhodes J. Fungal endophthalmitis following intraocular lens implantation. Am J Ophthalmol. 1977 Jan;83(1):1-8.

12 Pettit TH, Olson RJ, Foos RY, Martin WJ. Fungal endophthalmitis following intraocular lens implantation. A surgical epidemic. Arch Ophthalmol. 1980 Jun;98(6):1025-39.

13 Tarkkanen A, Raivio V, Anttila VJ, Tommila P, Ralli R, Merenmies L, et al. Fungal endophthalmitis caused by Paecilomyces variotii following cataract surgery: a presumed operating room air-conditioning system contamination. Acta Ophthalmol Scand. 2004 Apr;82(2):232-5.

14 Berler DK, Alper MG. Scleral abscesses and ectasia caused by Pseudomonas aeruginosa. Ann Ophthalmol. 1982 Jul;14(7):665-7.

15 Narita A, Seguchi J, Shiraga F. Paecilomyces lilacinus-induced scleritis following bleb-associated endophthalmitis after trabeculectomy. Acta Med Okayama. 2015;69(5):313-8.

16 Chung PC, Lin HC, Hwang YS, Tsai YJ, Ngan KW, Huang SC, et al. Paecilomyces lilacinus scleritis with secondary keratitis. Cornea. 2007 Feb;26(2):232-4.

17 Martínez YN, Cavello I, Hours R, Cavalitto S, Castro GR. Immobilized keratinase and enrofloxacin loaded on pectin PVA cryogel patches for antimicrobial treatment. Bioresour Technol. 2013 Oct;145:280-4.

18 Luangsa-Ard J, Houbraken J, van Doorn T, Hong SB, Borman AM, Hywel-Jones NL, et al. Purpureocillium, a new genus for the medically important Paecilomyces lilacinus. FEMS Microbiol Lett. 2011 Aug;321(2):141-9.

19 Yoshida M, Yokokura S, Kunikata H, Takada N, Maruyama K, Toyokawa M, et al. Endophthalmitis associated with Purpureocillium lilacinum during infliximab treatment for surgically induced necrotizing scleritis, successfully treated with 27-gauge vitrectomy. Int Ophthalmol. 2018 Apr;38(2):841-7.

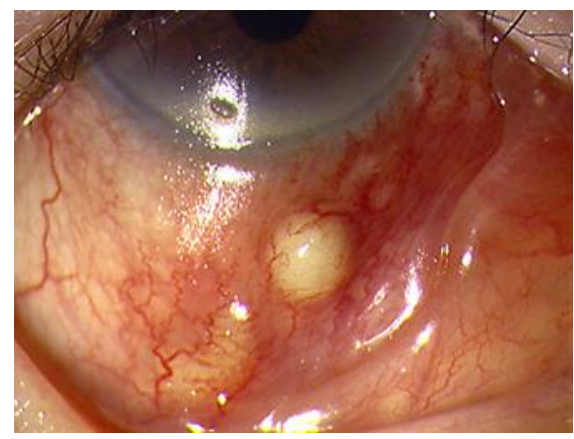

Fig. 1. Slit-lamp microscopy image of the patient's right eye obtained at the initial presentation. The conjunctival abscess and inflammatory anterior chamber cells in the inferonasal quadrant can be seen. 


\section{Case Reports in Ophthalmology}

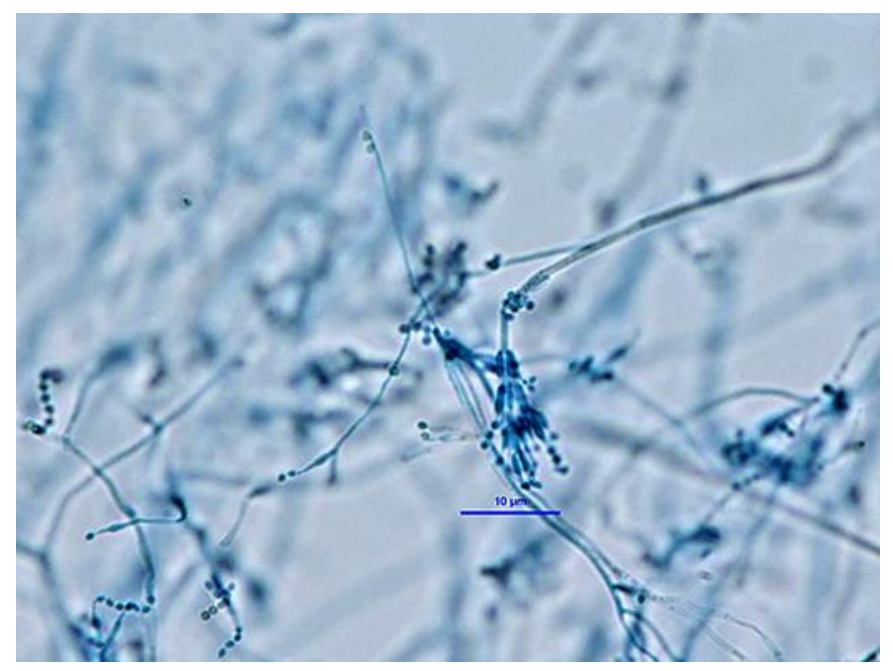

Fig. 2. PL culture on malt extract agar nutrient medium. PL was identified from its characteristic form. We found typical lilac sporulating colonies.

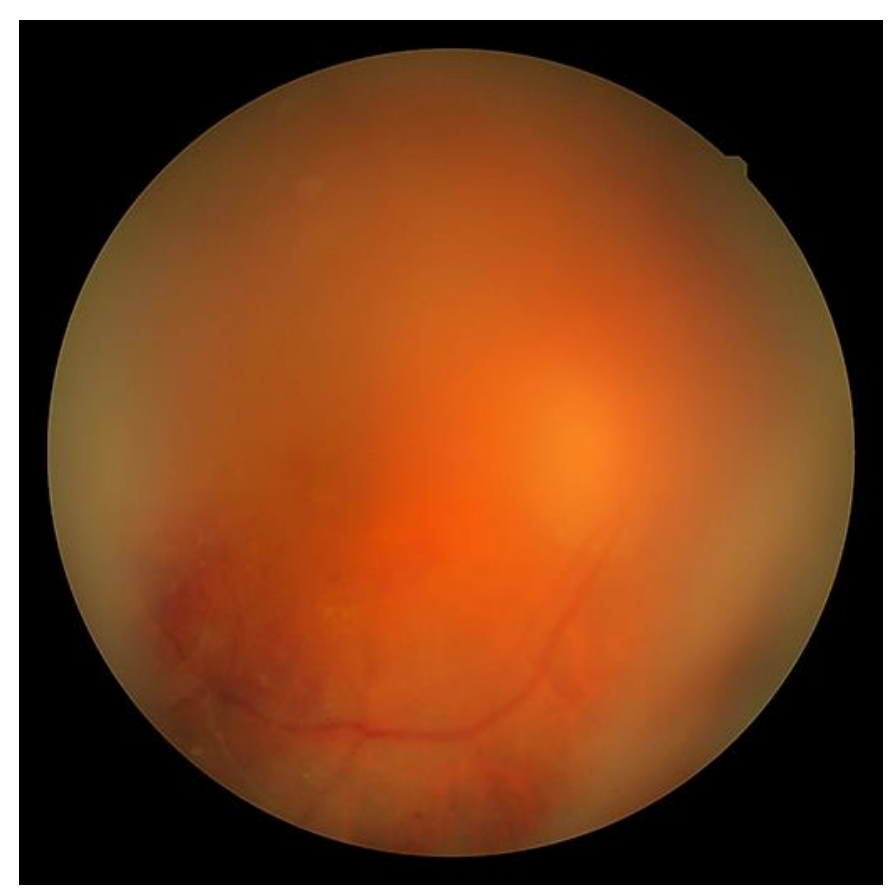

Fig. 3. Fundus image of the patient's right eye obtained 1 month after initial presentation and initiation of treatment, showing that the vitreous opacity had gradually worsened. 
Case Reports in Ophthalmology
Case Rep Ophthalmol 2020;11:256-262

DOI: $10.1159 / 000508389$

(c) 2020 The Author(s). Published by S. Karger AG, Basel www.karger.com/cop

Tara et al.: Fungal Endophthalmitis Caused by Paecilomyces lilacinus

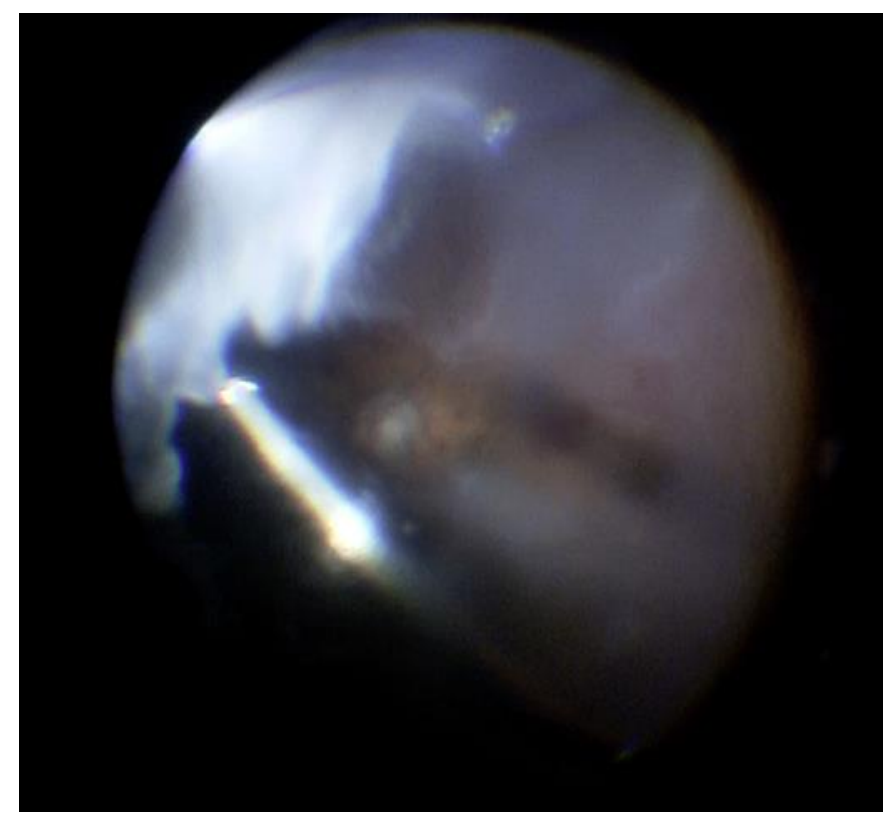

Fig. 4. Intraoperative image of the patient's right eye obtained during vitreous surgery. As the image shows, the vitreous opacity was severe mainly around the inferonasal quadrant and adjacent to the sclera at the site of the conjunctival abscess.

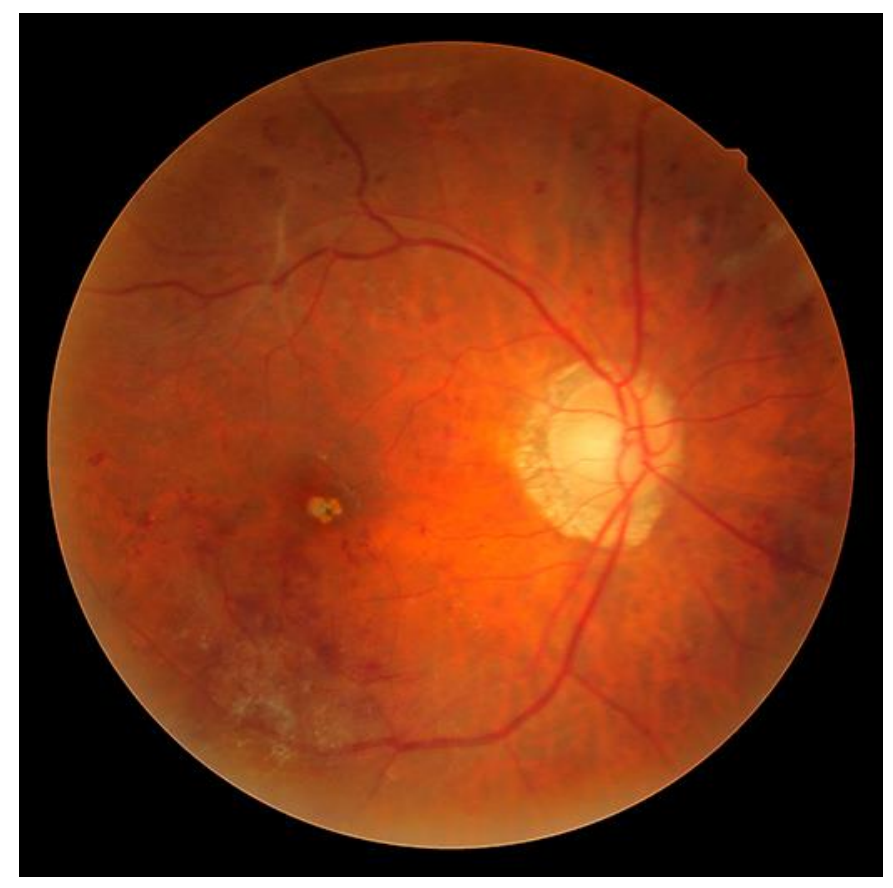

Fig. 5. Fundus image of the patient's right eye obtained after vitreous surgery. After surgery, the visibility of the ocular fundus recovered, and the corrected visual acuity was improved to 0.7 . 